This item was submitted to Loughborough's Research Repository by the author.

Items in Figshare are protected by copyright, with all rights reserved, unless otherwise indicated.

\title{
Monitoring aptamer-protein interactions using Tunable Resistive Pulse Sensing
}

PLEASE CITE THE PUBLISHED VERSION

http://dx.doi.org/10.1021/ac401764c

\section{PUBLISHER}

(c) American Chemical Society

\section{VERSION}

AM (Accepted Manuscript)

\section{PUBLISHER STATEMENT}

This work is made available according to the conditions of the Creative Commons Attribution-NonCommercialNoDerivatives 4.0 International (CC BY-NC-ND 4.0) licence. Full details of this licence are available at: https://creativecommons.org/licenses/by-nc-nd/4.0/

\section{LICENCE}

CC BY-NC-ND 4.0

\section{REPOSITORY RECORD}

Billinge, Emily R., Murray Broom, and Mark Platt. 2019. "Monitoring Aptamer-protein Interactions Using Tunable Resistive Pulse Sensing". figshare. https://hdl.handle.net/2134/16840. 


\title{
Monitoring Aptamer-Protein Interactions using Tunable Resistive Pulse Sensing.
}

\author{
Emily R. Billinge ${ }^{1}$, Murray Broom², Mark Platt ${ }^{1 *}$ \\ ${ }^{1}$ Department of Chemistry, Centre for Analytical Science, Loughborough University, \\ Loughborough, LE11 3TU, United Kingdom. \\ ${ }^{2}$ Izon Science Ltd., PO Box 39168, Burnside, Christchurch 8053, New Zealand. contact : \\ murray@izon.com
}

*Correspondence should be sent to m.platt@lboro.ac.uk 


\begin{abstract}
-
Aptamers are short single-stranded pieces of DNA or RNA capable of binding to analytes with specificity and high affinity. Due to their comparable selectivity, stability and cost, over the last two decades aptamers have started to challenge antibodies in their use on many technology platforms. The binding event often leads to changes in the aptamer's secondary and tertiary structure; monitoring such changes has led to the creation of many new analytical sensors. Here we demonstrate the use of a tunable resistive pulse sensing (TRPS) technology to monitor the interaction between several DNA aptamers and their target - thrombin. We immobilised the aptamers onto the surface of superparamagnetic beads, prior to their incubation with the thrombin protein. The protein binding to the aptamer caused a conformational change resulting in the shielding of the polyanion backbone; this was monitored by a change in the translocation time and pulse frequency of the particles traversing the pore. This signal was sensitive enough to allow the tagless detection of thrombin down to nanomolar levels. We further demonstrate the power of TRPS by performing real time detection and characterisation of the aptamer-target interaction and measuring the association rates of the thrombin protein to the aptamer sequences.
\end{abstract}

Key words - Thrombin, Aptamer, Resistive pulse sensing, superparamagnetic beads, association rate, biosensor. 


\section{Introduction -}

In recent years, developments in the field of diagnostics and biosensor technologies have been aided by the use of nanomaterials ${ }^{1-5}$. Synthesis and fabrication methods for nanoparticles in solution have evolved to the extent that particle size, shape, and composition can be easily modified. Technology platforms have also become more robust and reproducible to the extent that assays based upon nanomaterials offer significant advantages over conventional diagnostic systems with regard to assay sensitivity, selectivity, and practicality $^{6-13}$. Following the advancement of material synthesis, a range of new characterisation technologies capable of monitoring and measuring the size, surface chemistry, shape, optical, magnetic and even electrochemical properties have emerged ${ }^{14-20}$. Furthermore, with current synthesis strategies, materials can all be modified with a range of surface chemistries, patterning, and even functionalised with biomolecules ${ }^{21}$.

Within the fields of biosensors and diagnostics there is an increasing drive to reduce the time taken to yield a result from the point of sampling. With a desire to move towards point of care (POC) diagnostics, and facilitated by the miniaturisation of components, technologies previously used solely for particle characterisation are now being incorporated into sensor and diagnostic technology platforms. Light scattering ${ }^{19}$, fluorescence ${ }^{9,12,22}$, magnetoresistance and flow cytometry ${ }^{23,24}$ are now routinely used for particle characterisation as well as the detection of cells, proteins, and genomic and metabolomic analytes.

Each technology carries its own advantages and limitations regarding sensitivity, the ability to multiplex, biocompatibility and assay time. Current bioassays, such as those done by microscopy, are often inaccessible in resource-poor areas due to their running and maintenance costs, and need for skilled operators ${ }^{25}$. In recent years, nanopore systems utilising Coulter technology have seen resurgence, allowing the characterisation of colloid and nanoparticle based systems ${ }^{26}$. Known collectively as resistive pulse sensing (RPS), they offer an attractive technology format because the measurements provide information on individual particles within their natural environment ${ }^{14,27-30}$. RPS has been used to study numerous types of particles in different contexts with impressive resolution, including single molecule biophysics, protein sensing, biological detection ${ }^{27,28,30}$ and synthetic nanoparticle characterisation $^{31,32}$. A recent adaptation to RPS termed tunable resistive pulse sensing (TRPS) $)^{17,30}$ uses a conical tunable elastomeric pore allowing further versatility as the pore can be stretched in real time to suit the sample ${ }^{15}$. TRPS has been validated to accurately determine the concentration ${ }^{31}$ size $^{32}$ and surface charge of dispersed inorganic particles. The technique is a relatively inexpensive off the shelf instrument. The only consumable is the 
tunable pore, thus eliminating the need for a carrier gas, fluidics, or optics. Previously, TRPS has been utilised to monitor the aggregation of a special class of nanomaterial, termed nanorods, in the presence of analytes ${ }^{16}$, and the aggregation of gold nanoparticles in the presence or absence of DNA to detect single nucleotide polymorphisms ${ }^{33}$.

The interaction between an analyte and the particle is key to providing a selective and sensitive assay format. Where previously antibodies have been the capture probe of choice, aptamer technologies are gaining interest ${ }^{34-37}$. Aptamers are powerful reagents that bind target ligands with affinities comparable to antibodies. Aptamers are conventionally generated through the process known as SELEX ${ }^{38,39}$ (systematic evolution of ligands by exponential enrichment), where strong binding sequences are evolved/enriched from extensive libraries, or by CLADE ${ }^{40-43}$ (closed loop aptameric directed evolution) which produces the aptamers 'on-chip'. During the CLADE cycle the selection, modification and evolution of the DNA sequences are carried out in silico using a genetic algorithm (GA) to optimise the binding strength $^{42,43}$.

The modification of particle surfaces with aptamers should be a process well suited to RPS technologies. Information on size and surface charge can be measured simultaneously by analysing the pulse observed as a particle traverses the pore, displayed schematically in figure $1 \mathrm{~A}$.

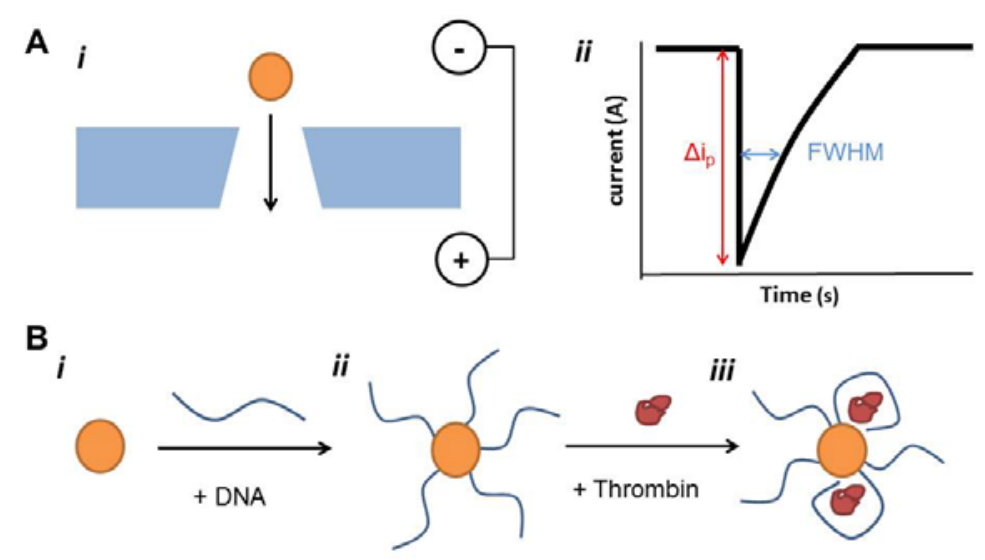

Figure 1: A (i) Schematic representation of a particle traversing the pore opening. (ii); Schematic representation of a pulse that occurs as the particle traverses the opening. The blockade magnitude $\left(\Delta i_{p}\right)$, is used to determine individual particle size; full width half maximum (FWHM) gives an indication of the time taken to traverse the pore. B Schematic of the experimental process, (i-ii) a bead functionalised with streptavidin is incubated with biotin tagged DNA, (iii) After removing any unbound DNA from the solution, the target protein is added to the sample.

A stable ionic current is established upon the application of an applied potential, and as the particles enter the pore orifice there is a measurable increase in resistance; the 
occlusion of ions results in a transient decrease in current. The conical pore gives rise to an asymmetric current pulse as resistance diminishes toward the base of the pore ${ }^{44}$.

For a conical pore, the change in resistance, $\Delta R$, across the length of the pore, $L$, is given by equation $1^{45}$,

$\Delta R=\rho \int_{0}^{L} \frac{d z}{A(z)}-R$

where $\rho$ is the resistivity of the electrolyte filling the pore, $A(z)$ is the cross sectional area perpendicular to the pore axis $z$, and $\mathrm{R}$ is the pore resistance when no blockage is present given by equation $2^{45}$,

$R=\frac{4 L \rho}{\pi D_{L} D_{S}}$

where $D_{L}$ and $D_{s}$ are the large and small pore diameters. The size of any biological or inorganic particle that traverses the pore, can be determined by measuring the change in resistance. Heins et al's work used this relationship to monitor and model the translocation events of small molecules ${ }^{45}$. Kozal et al recently derived a similar relationship between the change in resistance within a conical pore using an elastic pore sensor, which closely predicts the $\Delta R$ with nanoparticles traversing the pore ${ }^{46}$. Conical nanopores are of particular interest due to the localization of a narrow sensing zone at the small pore orifice, and modeling the pulse shape and magnitude within such pores is nontrivial ${ }^{44,47-49}$. The frequency of the pulses $J$, can be related to the concentration of the analyte, $C_{s}$, as well as the velocity of the traversing particle, $v_{p}$.

The velocity term is the sum of the fluidic, $v_{F}$ electrophoretic, $v_{E}$, and electroosmotic, $v_{O}$, velocities i.e. $v p=v_{F}+v_{E}+v_{O}$. Here we ignore the contribution from diffusion due to the magnitude of other forces ${ }^{46}$ and end effects are not taken into account in the analysis ${ }^{44} \cdot v_{p}$ can be written as $^{50}$;

$v_{p}=\frac{Q}{\pi\left(\frac{D_{S}}{2}\right)^{2}}+\frac{\epsilon \zeta_{\text {particle }}}{\eta} E-\frac{\varepsilon \zeta_{\text {pore }}}{\eta} E$ 
Where $Q=\frac{3 \pi D_{S}^{3} \Delta P}{128 \eta\left(\frac{L}{D_{L}-D_{S}}\right)}$

$\varepsilon$ and $\eta$ is the permittivity and kinematic viscosity, $\Delta P$ is the pressure across the pore, $\zeta_{\text {pore }}$ and $\zeta_{\text {particle }}$ is the zeta potential of the channel surface and particle respectively, $E$ is the electric field. The pulse frequency, $J$, is then related to both the velocity and the particle concentration $C_{s}$, via the equation ${ }^{44,51} ; J=C_{s} \times v_{p}$. For the system used in this study it has been demonstrated that the forces of electrophoresis, electro-osmosis and pressure are usually dominant $^{15,17,31,32,52}$. Therefore, if the pressure, ionic strength and composition of the buffer do not change during the experiments and the pore surface charge remains constant, any observed differences can be inferred to be due to the electrophoretic mobility. Particles that have been modified with strands of DNA should result in a change in particle frequency and duration over unmodified beads as the electrophoretic mobility is altered. In addition, the RPS technology should be able to monitor the interaction between the DNA and any analyte that binds to it, as such interactions should result in a conformational change and shielding of the DNA charge.

Here we demonstrate the use of TRPS technology to observe the interaction between aptamer modified superparamagnetic particles with their target protein, thrombin. Thrombin was chosen as it is one of the first and most widely studied aptamers, and its binding mechanism is well characterised and understood ${ }^{38,53,54}$. Whilst thrombin itself is an important biomarker and plays a role in the coagulation cascade and cardiovascular disorders ${ }^{55}$, there are many methods and technology platforms for its quantification, and it is often used to validate emerging aptamer technologies and hence its use here ${ }^{56}$. In the presence of thrombin, aptamer sequences containing a "GGNNGGNGNGGNNGG" (where $\mathrm{N}$ represents $\mathrm{T}$ or $\mathrm{A}$ ) motif undergo a conformational change to form a G-quadruplex structure ${ }^{57}$. As it possesses such a well-studied structure, it also allows us to use modified thrombin aptamer sequences where we deliberately control and modify the binding shape to study the effects upon the signal in the TRPS ${ }^{43,53}$.

The immobilisation of the DNA onto the particle surface is confirmed by changes in the observed particle count rate and decrease in translocation times. These results confirm the change in surface charge from a relatively neutral to highly negative surface. The incubation of the thrombin target with the beads causes a shielding effect, where the thrombin protein masks the negative charge and the particle count rate and translocation times change. This 
interaction forms the basis of a real time sensor with limits of detection down to nanomolar levels of thrombin with assay times of 3 minutes. The sensitivity of the TRPS allows us to monitor the binding kinetics of three different thrombin aptamers. Using this technique we are able to calculate a dissociation constant for the particle-thrombin reaction on the order of $39 \times 10^{-9} \mathrm{M}$.

\section{Methods -}

\section{Chemicals and reagents}

The following chemicals were sourced from Sigma Aldrich, United Kingdom, without any further purification unless otherwise stated: phosphate buffered saline (PBS - P4417), Tween 20 (P1379), Bovine Serum Albumin (BSA - A2153), and thrombin (T7513). DNA sequences were purchased as lyophilised powders with HPLC purification from Entelchon (Germany) : 5’GGT TGG TGT GGT TGG TTT TTT TTT T-Biotin-3’ (Thrombin-15) and 5’TGG GAG TAG GTT GGT GTG GTT GGG GCT CCC CTT TTT-Biotin-3’ (Thrombin-MArray). The sequences 5’GGT TGG TTT ATT TTA CTA GTG GCC AGG-Biotin-3’ (ThrombinEvol) and a random biotin-tagged 10mer were purchased as lyophilised powders with HPLC purification from Sigma-Aldrich (UK). These were made up to a stock concentration of $100 \mathrm{pmol} / \mu \mathrm{L}$ with deionised water.

\section{Particles}

Streptavidin modified superparamagnetic particles of 128nm diameter were purchased from Ademtech (France). Carboxyl beads of known concentration and diameter (203 nm) were sourced from Izon Science (Christchurch, New Zealand) and used as a calibrant at a concentration of $\left(4 \times 10^{9}\right.$ particles $\left./ \mathrm{mL}\right)$. Water purified to a resistivity of $18.2 \mathrm{M} \Omega \mathrm{cm}$ (Maxima) was used to make all solutions unless otherwise specified. In all experiments $1 \times$ PBST (0.05\% Tween) was used as the buffer.

\section{Binding assays}

Immobilisation of DNA onto the beads

$128 \mathrm{~nm}$ streptavidin coated beads were diluted to a concentration of approximately $3 \times 10^{9}$ particles/mL; this stock was vortexed and sonicated to ensure monodispersity. To $40 \mu \mathrm{L}$ of beads a further $10 \mu \mathrm{L}$ of varied ratios of PBST and aptamer dilutions was added to provide a range of final concentrations from $0.1 \mathrm{nM}$ up to $10000 \mathrm{nM}$ of DNA. These were vortexed and placed on a rotary wheel for 30 minutes at room temperature prior to analysis.

Thrombin Binding Assay: Bead preparation: 
Step 1 - attachment of aptamer onto the beads

$128 \mathrm{~nm}$ beads were diluted in PBST to a concentration of approximately $3 \times 10^{9}$ particles/mL. A ten-fold excess of biotinylated DNA (according to the binding capacity of the supplier) was added to the beads and incubated at room temperature for 30 minutes. In control experiments the same concentration of a random 10mer was added and the same procedure followed.

Step 2 - removal of excess DNA

The sample was centrifuged for 2 minutes at $10000 \mathrm{rpm}$ and immediately placed in a MagRack (Life Sciences). After 5 minutes the beads had formed a visible cluster in the sample vial adjacent to the magnet and the solution was carefully removed and replaced with an equal volume of PBST. This wash stage was performed twice.

Step $3 a-$ Thrombin concentration investigation

A variety of concentrations of thrombin protein was added to the beads which were then incubated on a rotary wheel for 30 minutes before analysis, these ranged from a final concentration of $0.1 \mathrm{nM}-10000 \mathrm{nM}$. In these experiments the concentration of the beads was kept constant.

Step $3 b$ - real time binding experiments

$36 \mu \mathrm{L}$ of washed bead-aptamer sample, described in step 2, was pipetted into the upper fluid cell of the TRPS instrument, this dispersion was recorded for $60 \mathrm{~s}$ at which time the software was paused and $4 \mu \mathrm{L}$ of the required test solution was added into the solution. Recording was immediately resumed. Data was then captured for a minimum of 60 seconds and included a a minimum of 50 data points. The addition of the $4 \mu \mathrm{l}$ of solution required the pipette tip to be placed into the liquid, the $4 \mu \mathrm{l}$ was then added and the tip removed. Other than the convection caused by the removal of the tip no additional stirring was induced.

\section{Tunable Resistive Pulse Sensing (TRPS)}

Measurements were made using the qNano system obtained from Izon Science (Christchurch) incorporating the fluid cell, stretching apparatus, data recording and analysis software (v.2.2). The pores used were designated 'NP200' by the manufacturer, and are described as most suitable for detecting particles in the range $100-300 \mathrm{~nm}$. The macroscopic stretch applied to the membrane is given in each figure legend. An appropriate voltage (circa $\sim 0.36 \mathrm{~V}$ ) was selected for all experiments to enable the detection of the peaks above the level of noise $(<10 \mathrm{pA})$. This was judged to be adequate when the peak magnitude 
was greater than $0.12 \mathrm{nA}$. The pore stretch and applied voltage was the same for similar sets of experiments to allow us to compare data. Typically a bandwidth filter of $1 \mathrm{kHz}$ was applied during measurements, and modal values from data histograms are used in all plots. In all experiments $80 \mu \mathrm{L}$ of electrolyte buffer was placed in the lower fluid cell. The volume in the upper fluid cell was typically $40 \mu \mathrm{L}$ unless experiments were being performed where a protein solution was added into the cell after 60 seconds. In this case the initial volume was $36 \mu l$ in the upper fluid cell and after 60 seconds $4 \mu \mathrm{l}$ of the solution was added giving a total volume of $40 \mu \mathrm{l}$; after recording, this solution was withdrawn and the fluid cell and pore washed by repeatedly replacing PBST in the upper fluid cell until no particles were apparent on the signal trace. To generate error bars in all figures the mean and standard deviation of repeat experiments was used.

\section{Results and discussion -}

\section{Binding of biotinylated DNA to the Streptavidin beads}

The first experiment modified the streptavidin coated beads with the biotinylated-Thrombin15, displayed schematically in figure 1B. A tenfold excess of DNA over the known binding capacity (final concentration of $171 \mathrm{nM}$ ) was added to ensure all the free binding sites were occupied. The beads were incubated at room temp for 30 minutes, before placing the sample into the TRPS instrument. During each experiment, the applied voltage always had a positive bias applied to the electrode underneath the membrane i.e. on the opposite to where the sample was placed, this orientation makes it easier to observe negatively charged beads ${ }^{3}$. We noted two distinct changes in the signal upon the binding of Thrombin-15 aptamer to the beads. The first was an increase in particle count rate from circa 100 to 400 beads per minute, and the second was a decrease in the FWHM values, from $1.2 \mathrm{~ms}$ to $0.3 \mathrm{~ms}$, for the streptavidin and DNA coated beads respectively, and these trends were expected. In the setup used here the sample is placed into an upper fluid cell, and whilst the peak duration can be governed by pressure-driven flow in the present setup, the increase in electrophoretic mobility can clearly be observed. To demonstrate that this effect was dependent upon the quantity of DNA on the beads surface, the concentration of DNA was varied and the results are shown in, figure 2. 


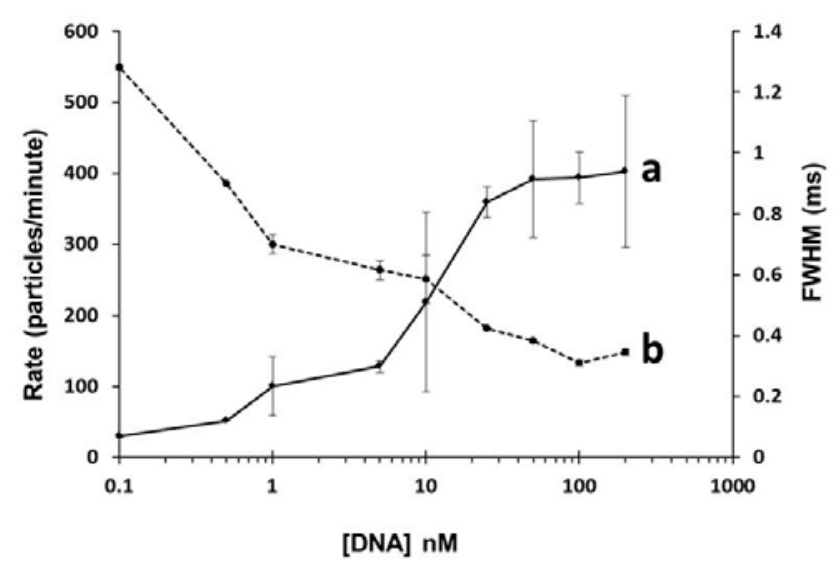

Figure 2: Scatter plot displaying average rate (a) mode FWHM (b) against concentration of Thrombin-15 using a concentration of beads of $3 \times 10^{9}$ particles $/ \mathrm{mL}$. A "NP200" pore with a membrane stretch of $44.50 \mathrm{~mm}$, voltage of $0.4 \mathrm{~V}$, and $40 \mu \mathrm{l}$ of sample in the upper fluid cell was used. Each data point represents the average value from four experiments and error bars represent 1 standard deviation from the mean.

As the concentration of DNA increases, as did the coverage of DNA on the beads surface, the FWHM and particle rate decrease and increase respectively until a consistent value is obtained of around $\sim 400$ particles/min and $0.28 \mathrm{~ms}$. In these experiments we failed to observe any increase in $\Delta i_{p}$, figure-S1, upon the binding of the DNA. This is most likely attributed to the low sensitivity of the pore system under the current stretch and voltage conditions used here to resolve these small changes in size. We repeated this experiment with a random biotinylated 10-mer, results shown in figure-S2. Whilst similar trends were observed we noted that the absolute change in values for both the particle rate and FWHM were smaller (100 to 250 beads per minute and $0.9 \mathrm{~ms}$ to $0.5 \mathrm{~ms}$, respectively); this is not unexpected as the shorter sequence would produce a smaller charge density on the beads surface, therefore having a less profound effect on translocation events.

\section{Incubation of aptamer modified beads with thrombin protein -}

Having modified the streptavidin beads with the Thrombin-15 aptamer, we removed any unbound aptamer from solution by magnetically extracting the beads and suspending them in fresh buffer. The aptamer modified beads were then incubated with the thrombin protein for 30 minutes, before $40 \mu \mathrm{l}$ of sample was analysed in the TRPS. A range of concentrations of thrombin were used; figure 3A and 3B display plots of particle rate and FWHM values, respectively, for the Thrombin-15 (line a, solid red). As we increase the concentration of the protein the particle rate and FWHM values decrease and increase respectively. We attribute this to the protein binding to the aptamer and shielding some of the negative charge, shown schematically in figure $3 \mathrm{Ci}$. At the higher concentrations of thrombin $>10 \mathrm{nM}$, further binding 
of the thrombin protein cannot be observed using the particles rate as it remains relatively constant. However, the FWHM for thrombin 15, as displayed in figure $3 \mathrm{~B}$, does continue to rise. We have calculated that at concentrations of thrombin over $15 \mathrm{nM}$, there are circa 2 aptamers for every thrombin molecule, leading us to conclude that steric hindrance, rather than binding site saturation, is responsible for this. Plotted in figure-S3 is the relative size of the blockade events versus thrombin concentration, whilst thrombin may contain two epitopes, unlike previous studies here we do not observe any aggregation of the magnetic beads $^{58,59}$. As a control we carried out the same experiment using a BSA protein, displayed in figure 3 (red dashed line - curve c). The particle count rate and FWHM remain constant across the entire concentration range of BSA, illustrating that the aptamer is specific to the thrombin target. This is an expected result given the depth of work using the sequence, but also verifies that the observed trends are unrelated to nonspecific interaction between the protein and DNA or surface of the beads.
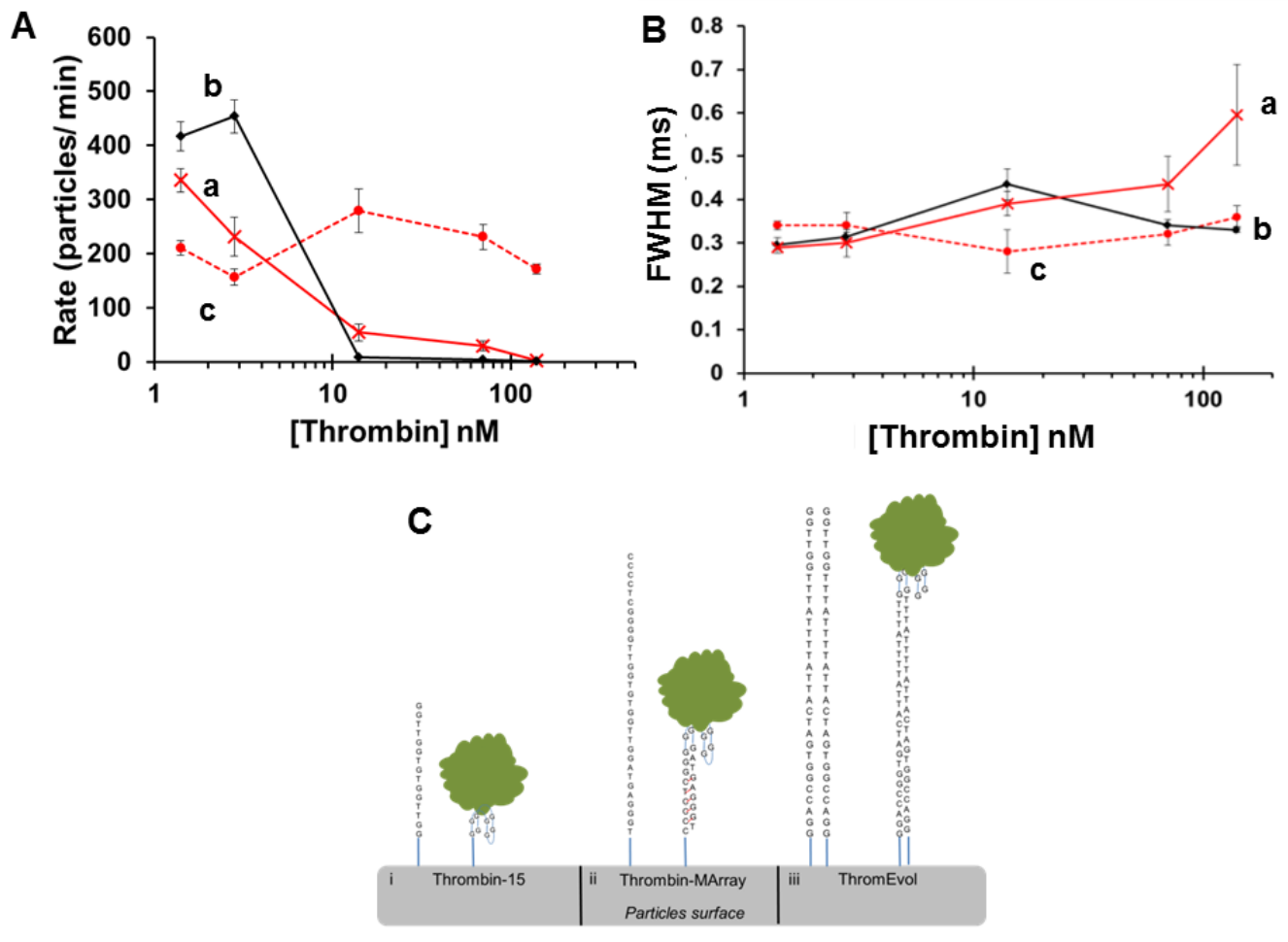

Figure 3: A Scatter plot displaying the effect of increasing concentrations of thrombin or BSA on rate and FWHM (inset) $\mathbf{a}=$ Thrombin-15, $\mathbf{b}=$ ThrombinMArray, $\mathbf{c}=$ BSA control. A "NP200" pore with a membrane stretch of $44.50 \mathrm{~mm}$, voltage of $0.36 \mathrm{~V}$, and $40 \mu \mathrm{l}$ of sample in the upper fluid cell was used. Each data point represents the average value from four experiments and error bars represent 1 standard deviation from the mean. B Schematic of the thrombin protein binding to the three different aptamer sequences.

We also tested a second thrombin aptamer sequence, termed Thrombin-MArray, plotted in figure 3 (black - curve b). This sequence was previously optimised from one of the 
original SELEX aptamers on a DNA microarray surface using a genetic algorithm ${ }^{53}$. The sequence contains the standard G-quadruplex, but also has complimentary strands of DNA flanking the quadruplex structure, forming a structure stabilising duplex. The measured affinity using SPR analysis for the Thrombin-MArray aptamer was comparable to the original Thrombin- $15^{53}$. We chose this sequence as a comparison because research has shown that in some cases modifications of the original 15mer can in fact enhance the binding on surfaces $^{53,60}$, leading to a greater sensitivity.

The difference in the mechanism for binding thrombin between the two aptamers lies in the existence of the complimentary region in the Thrombin-MArray aptamer. In order to form a stable structure the sequences must fold in half, stabilised by the binding of the complementary regions, shown schematically in figure 3Cii. A plot of particle rate and FWHM versus thrombin concentration is shown in figure 3B (c - black line). Whilst we observe similar trends to those with the original $15 \mathrm{mer}$, i.e. the particle rate drops at higher concentrations of thrombin, we noted that at lower concentrations of protein target very little deviation in the particle rate and FWHM was recorded. In addition, the total change in FWHM across the concentration range was much smaller, 0.29 to $0.34 \mathrm{~ms}$.

Again a control experiment using BSA protein was performed for this aptamer and the data are shown in figure-S4. There are two observations here worth discussing, the decrease in sensitivity, i.e. no observed a change in particle rate of FWHM at concentrations lower than $10 \mathrm{nM}$, and the small change in FWHM across the full range of thrombin concentrations for the Thrombin-MArray sequence.

Both aptamer sequences have been previously characterised using standard surface plasmon resonance methods, and yielded similar, $K_{D}$ values. Upon scrutiny the reason for the deviations in behaviour may be attributed to the mechanism of binding. The binding of the protein to the Thrombin-MArray aptamer, shown in figure 3Bii, requires the DNA to fold back and form complementary base pairs near the particle surface, which stabilises the structure and allows the free lysine residue on the protein to bind to the G-quadruplex. In this orientation the thrombin protein is held close to the surface of the bead and neighbouring aptamer strands that are unbound extend out into solution. At low protein coverage, the dominant effect on the particle rate and FWHM is the protrusion of the phosphate backbone from the DNA past the protein, and as the coverage of thrombin on the surface increases and more aptamer strands are masked, the particle rate decreases.

In both sets of experiments, it is unlikely that the number of bound proteins on the surface equals the total number of aptamers due to steric hindrance. The duration of 
translocation, which can be inferred by FWHM, of the Thrombin-MArray beads at concentrations of thrombin $>10 \mathrm{nM}$ remains lower than the Thrombin-15 indicating a higher negatively charged surface still exists, this we propose is due to the longer sequence and protrusion of the DNA past the bound protein. Because the change in particle rate was always easier to observe and more reproducible than the FWHM, we only show and use the rate data here after.

\section{Measuring the association rate -}

The above measurements were recorded after a 30 minute incubation period between the beads and target protein; this should be a sufficient time for the binding between the protein and aptamer to have occurred. Calculations based upon Smoluchowski theory ${ }^{61,62}$ suggest that the reaction should proceed quickly under the conditions and concentrations used here and binding between the protein and aptamer should occur within seconds. To ascertain if we could observe the binding to the beads in real time we placed $36 \mu \mathrm{l}$ Thrombin -15 modified beads into the upper fluid cell; we collected data for $60 \mathrm{~s}$ to obtain a base particle rate, the data collection was paused whilst the thrombin protein was added to the upper fluid cell, before continuing data collection. The change in particle rate as a function of time, for four experiments repeated under the same thrombin concentration are displayed in figure 4A. To demonstrate that the addition of extra solution itself does not cause a significant change in particle count rate, the same volume of PBST without Thrombin was added into the upper fluid cell, data from repeat experiments shown in figure-S5 and table-S1. Typically the addition of the extra $4 \mu \mathrm{l}$ of solution to the upper fluid cell caused a small deviation in the gradients of the line, on average the gradient of the line changed by around $-4 \%$ upon the addition of the solution indicating a decrease in particle count rate. The particle rate was calculated at each data point using the equation-S1. 

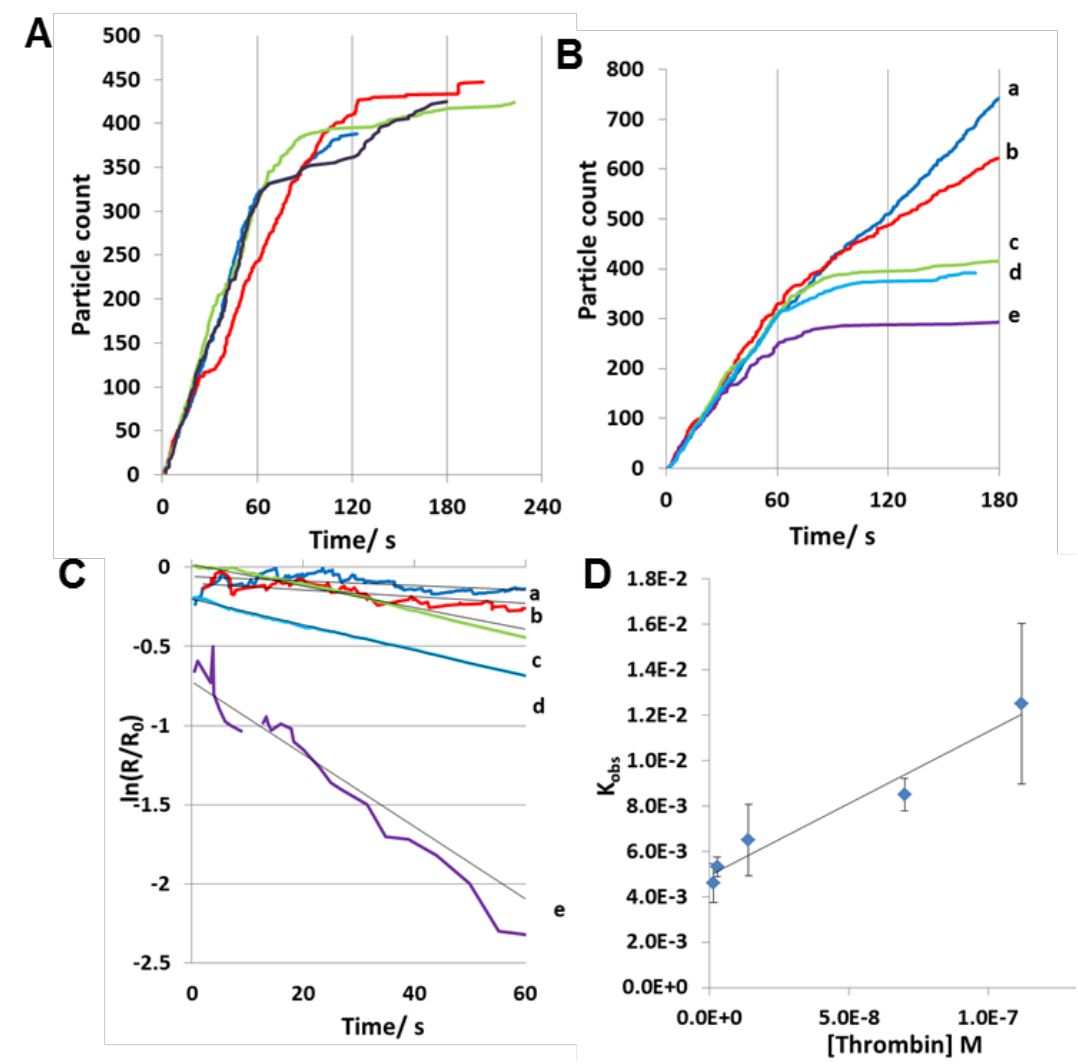

Figure 4: A, Replica experiments of real time particle rate measurements for Thrombin-15 modified beads. At $t$ $=036 \mu \mathrm{l}$ of sample was present in the upper fluid cell, at $\mathrm{t}=60 \mathrm{~s} 4 \mu \mathrm{L}$ of Thrombin solution was added giving a final concentration of $15 \mathrm{nM}$. B, One set of experiment demonstrating the change in rate with respect to the concentration of thrombin. At $t=60 \mathrm{~s} 4 \mu \mathrm{L}$ of Thrombin solution was added giving a final concentration of thrombin (navy blue) $=1.5 \mathrm{nM}, \mathrm{b}$ (red) $=3 \mathrm{nM}, \mathrm{c}$ (green) $=15 \mathrm{nM}, \mathrm{d}$ (blue) $=77 \mathrm{nM}$, e (purple) $=154 \mathrm{nM}$. C, Plot of rate of change in particle count, $\ln \left(R / R_{0}\right)$, versus time, for the experiment shown in part B. $t=0$ indicates the point at which thrombin was added to the particle solution. $D$, Plot of $K_{\text {obs }}$ versus concentration of thrombin. The values represent the mean from four experiments. The data was obtained using a "NP200" pore with a membrane stretch of $44.50 \mathrm{~mm}$, an applied voltage of $0.4 \mathrm{~V}$.

After the addition of the protein into the fluid cell we observed a decrease in particle count until a continuous value is obtained after approximately $120 \mathrm{~s}$. The observed change in particle count is attributed to the same effect described above: the binding of the protein to the surface and the masking of the negative DNA backbone. As presented in figure 4A, the observed effects were reproducible over several measurements. Figure 4B illustrates the change in particle rate as a function of thrombin concentration, as the concentration of protein increases, the magnitude of the change and the speed of the change also increases. Plot e appears to deviate and change its rate prior to the additions of the protein. Upon inspection of the trace it appears that a blockage i.e. a period of time where no particles traversed the pore, occurred at $34 \mathrm{~s}$, which lasted approx. $4 \mathrm{~s}$, after this time the rate returned to a steady value. We show only one of triplicate measurements in figure 4b; figure-S6 shows the repeat measurements with the blockage highlighted. We also show the analysis of the particle rate 
before and after the blockage in figure-S6c. In calculating the rates of reactions below we used the average value from all three measurements.

As the thrombin protein is in excess of the beads concentration by several orders of magnitude, we assumed a first order reaction where a plot of the rate of change, $\ln \left(R / R_{0}\right)$, versus time should yield a straight line with gradient of $\mathrm{k}_{\mathrm{obs}}$. Here, $R$ is used to represent instantaneous rate in a first-order rate equation. $\mathrm{R}$ was calculated by dividing equation-S1 by 60 , and $R_{0}$ is average rate of particles per second, recorded in the 60 seconds prior to the injection of the protein and $\mathrm{K}_{\mathrm{obs}}=\mathrm{k}_{\mathrm{a}}$ [Thrombin] $+\mathrm{k}_{\mathrm{d}}$. Thus a plot of $\mathrm{K}_{\mathrm{obs}}$ versus concentration of thrombin should yield a straight line with the gradient of $k_{a}$ and an intercept of $k_{d}$. Figure 4C shows the rate of change versus time for five different thrombin concentrations, figure-S7 illustrates the same analysis applied to the data given in figure 4A to allow a comparison of the reproducibility of the procedure. In the calculations a minimum of $60 \mathrm{~s}$ was collected after the injection of the protein to calculate the reaction rates.

The gradients from each experiment are plotted as a function of concentration in figure 4D and from the regression line $\mathrm{k}_{\mathrm{a}}$ is calculated to be $7.42 \times 10^{4} \mathrm{M}^{-1} \mathrm{~s}^{-1}, \mathrm{k}_{\mathrm{d}} 2.9 \times 10^{-3} \mathrm{~s}^{-1}$ and gives a $K_{D} 39.1 \mathrm{nM}$, which is comparable to measurements reported in the literature of 6 $\mathrm{nM}^{63}, 20 \mathrm{nM}^{59}, 200 \mathrm{nM}^{64}, 102.6 \mathrm{nM}^{65}$, and 75-100 nM $\mathrm{M}^{66}$. We performed the same set of experiments for the Thrombin-MArray aptamer and obtained a $k_{a}$ of $4.03 \times 10^{4} \mathrm{M}^{-1} \mathrm{~s}^{-1}, \mathrm{k}_{\mathrm{d}}$ $3.40 \times 10^{-3} \mathrm{~s}^{-1}$ and $\mathrm{K}_{\mathrm{D}} 84.3 \mathrm{nM}$. This compares favourably to the value obtained by the authors using surface plasmon resonance techniques, SPR, of $28 \mathrm{nM}^{53}$. We noted again that when a low $(<10 \mathrm{nM})$ concentration of thrombin was used we failed to see a change in signal for the Thrombin-MArray aptamer. Figure 5A, curve a, illustrates the particle rate for the Thrombin-MArray, and curve c, the Thrombin-15, after the injection of the thrombin protein at $\mathrm{t}=60 \mathrm{~s}$, we fail to see any change in the rate for the Thrombin-MArray aptamer. This observation agrees with the data displayed in figure 3.

We hypothesised that the difference in the observations for the two aptamers, which have similar $k_{a}$ and $k_{d}$ values from traditional SPR methods, is due to mechanistic effects, i.e. the protein is buried within a negative DNA layer, or the hindrance of the protein binding to the Thrombin-MArray aptamer on a beads surface rotating at $\sim 400 \mathrm{~s}^{-1}$. As we increase the concentration of the protein in figure 5B - D from $15 \mathrm{nM}$ to $154 \mathrm{nM}$, we observe the rate of change for the Thrombin-MArray starts to increase and eventually matches that of the Thrombin-15. Three control experiments are shown in figure S-9, in curve 1 BSA protein is added to the cell containing Thrombin-15 modified beads, curve 2 thrombin is added to carboxyl coated polystyrene beads, and curve 3, thrombin is added to a superparamagnetic 
bead modified with a random 10mer. In each experiment no deviation or change in particle rate after the additional of the protein was observed.
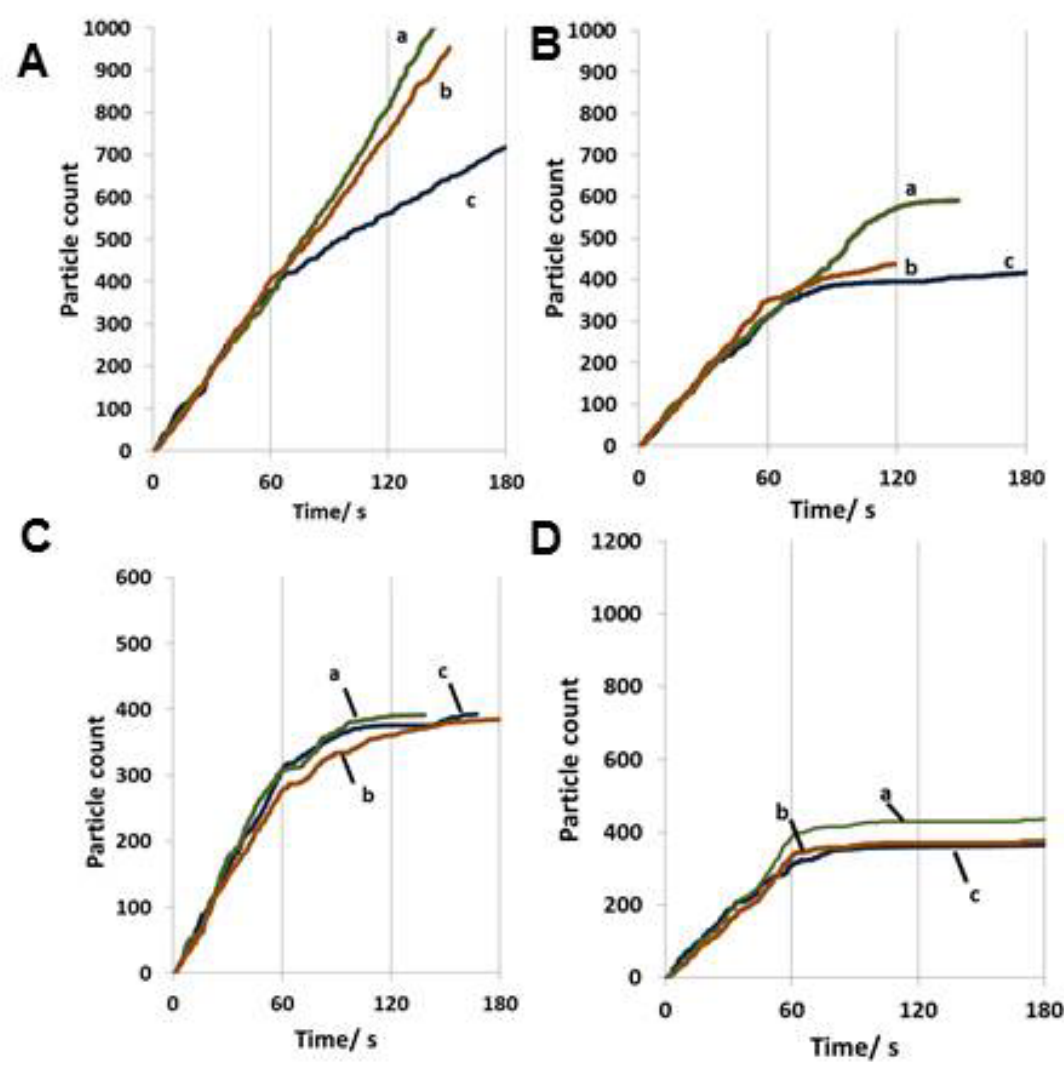

Figure 5: Real time particle rate measurements taken for three aptamers, curves are labelled a, b and c, representing data from Thrombin-MArray, ThromEvol and Thrombin-15 respectively. At $t=036 \mu$ of sample was present in the upper fluid cell. $A, 4 \mu$ of thrombin solution added at $t=60 \mathrm{~s}$ giving a concentration of 3 $\mathrm{nM}$. $B, 4 \mu \mathrm{l}$ of thrombin solution added at $t=60 \mathrm{~s}$ giving a concentration of $15 \mathrm{nM}$. $C, 4 \mu \mathrm{l}$ of thrombin solution added at $t=60 \mathrm{~s}$ giving a concentration of $45 \mathrm{nM}$. $D, 4 \mu \mathrm{l}$ of thrombin solution added at $t=60 \mathrm{~s}$ giving a concentration of $154 \mathrm{nM}$. The data was obtained using a "NP200" pore with a membrane stretch of 44.50mm, an applied voltage of $0.4 \mathrm{~V}$. Control experiments are represented in figure S-9.

We tested a third aptamer, termed ThrombinEvol, which was raised via the CLADE technique $^{43}$. This sequences does not contain the typical G-quadruplex motif but still possesses a reported $\mathrm{k}_{\mathrm{a}}$ of $8.7 \times 10^{4} \mathrm{M}^{-1} \mathrm{~s}^{-1}, \mathrm{k}_{\mathrm{d}} 2.04 \times 10^{-3} \mathrm{~s}^{-1}$ and $\mathrm{K}_{\mathrm{D}} 23.45 \mathrm{nM}^{43}$ when measured with SPR technologies. Importantly, this aptamer binds to the protein via the base pairs at the end of the sequences shown in figure 3Biii. This binding mechanism was hoped to lead to an improved sensitivity as unlike the Thrombin-MArray sequence the protein sits on top of the aptamer strands. The real time particle rate measurements for the sequences are presented in figure 5A - D (curves b). The ThrombinEvol sequence has an improved 
response at the lower protein concentrations and consistently performs better than the Thrombin-MArray, figure-S8 shows one set of data illustrating the change of rate across the full range of concentrations. The measured reaction rates based upon triplicate measurements for the Evol aptamer were $\mathrm{k}_{\mathrm{a}}$ of $5.78 \times 10^{4} \mathrm{M}^{-1} \mathrm{~s}^{-1}, \mathrm{k}_{\mathrm{d}} 4.80 \times 10^{-3} \mathrm{~s}^{-1}$, and $\mathrm{K}_{\mathrm{D}} 83.1 \mathrm{nM}$, see figure-S10 for the plot of $\mathrm{K}_{\mathrm{obs}}$ versus concentration. Whilst this suggests the shielding of the charge via the protein is key in observing a change in particle rate, the clearest response across the full range of the protein concentrations was the original Thrombin-15 aptamer. These results suggest that whilst shielding the charge on the DNA is an essential part of the signal, to obtain a strong change in particle rate a conformational change must also take place.

\section{Conclusions -}

Here we present the use of TRPS to monitor the changes in surface functionality on superparamagentic beads. We utilise this signal to study the interaction of DNA aptamers with their target protein. The changes in signal, namely particle rate and FWHM, are sensitive enough to monitor the immobilisation of DNA on to the beads surface, and then the binding of the protein to the aptamers. Our results demonstrate that TRPS is now one of many technologies capable of monitoring the interaction between aptamers and their target protein. We have demonstrated that TRPS allows for real time monitoring of the proteinaptamer interaction allowing the reaction rates to be calculated. This offers a new technique for monitoring the association rates of analytes directly on the beads surface without the need for identification labels such as fluorescence or utilising standard SPR technologies. The current method does have a limited working range, as at higher concentrations of target a reduced particle count rate makes obtaining a sufficient particle count difficult, evidence of this is in the increased size of the error bars at higher concentrations in figure 4D and S10. Work is underway in the laboratories to expand the dynamic range by varying the bead size and binding capacity of the surfaces.

\section{Acknowledgment}

The authors thank Dr D. Aubert, Dr C. Williams and Mr H. Van der Voorn for support. E Billinge and M. Platt thank Prof C. L. P Thomas support and advice, as well as the members of the Centre for Analytical Science. MP was funded by the European Commission for Research (PCIG11-GA-2012-321836 Nano4Bio), and Loughborough University Chemistry Department (Start-up fund). 


\section{Supporting Information}

Additional information as noted in text.

This material is available free of charge via the Internet at http://pubs.acs.org

\section{Conflict of interest}

MP and ERB declare no competing financial interest. M. B holds $<0.1 \%$ of shares within Izon Science Ltd 


\section{References}

(1) Oh, B.-K.; Park, S.; Millstone, J. E.; Lee, S. W.; Lee, K.-B.; Mirkin, C. A. Journal of the American Chemical Society 2006, 128, 11825.

(2) Elghanian, R.; Storhoff, J. J.; Mucic, R. C.; Letsinger, R. L.; Mirkin, C. A. Science 1997, $277,1078$.

(3) Wang, Y.; Kececi, K.; Mirkin, M. V.; Mani, V.; Sardesai, N.; Rusling, J. F. Chemical Science 2013, 4, 655.

(4) Rosi, N. L.; Mirkin, C. A. Chemical Reviews 2005, 105, 1547.

(5) Nam, J.; Thaxton, C.; Mirkin, C. Science 2003, 301, 1884

(6) Bardhan, R.; Lal, S.; Joshi, A.; Halas, N. J. Accounts of Chemical Research 2011, 44,

936.

(7) Farokhzad, O.; Cheng, J.; Teply, B.; Sherifi, I.; Jon, S.; Kantoff, P.; Richie, J.; Langer, R. Proc Natl Acad Sci USA 2006, 103, 6315

(8) Abe, M.; Suwa, T. Physical Review B 2004, 70, 235103.

(9) Bruchez, M., Jr.; Moronne, M.; Gin, P.; Weiss, S.; Alivisatos, A. P. Science 1998, 281,

2013.

(10) Whitesides, G. Nature Biotechnology 2003, 21, 1161

(11) Giri, S.; Sykes, E. A.; Jennings, T. L.; Chan, W. C. W. ACS Nano 2011, 5, 1580.

(12) Michalet, X.; Pinaud, F. F.; Bentolila, L. A.; Tsay, J. M.; Doose, S.; Li, J. J.; Sundaresan, G.; Wu, A. M.; Gambhir, S. S.; Weiss, S. Science 2005, 307, 538.

(13) Yu, C.; Irudayaraj, J. Analytical Chemistry 2006, 79, 572.

(14) Roberts, G. S.; Yu, S.; Zeng, Q.; Chan, L. C. L.; Anderson, W.; Colby, A. H.; Grinstaff, M. W.; Reid, S.; Vogel, R. Biosensors and Bioelectronics 2011.

(15) Roberts, G. S.; Kozak, D.; Anderson, W.; Broom, M. F.; Vogel, R.; Trau, M. Small 2010, $6,2653$.

(16) Platt, M.; Willmott, G. R.; Lee, G. U. Small 2012, 8, 2436.

(17) Kozak, D.; Anderson, W.; Vogel, R.; Trau, M. Nano Today, 6, 531.

(18) Myers, F. B.; Lee, L. P. Lab on a Chip 2008, 8, 2015.

(19) Mandy, F.; Gratama, J. W. Cytometry Part B: Clinical Cytometry 2009, 76B, 365.

(20) Prina-Mello, A.; Whelan, A. M.; Atzberger, A.; McCarthy, J. E.; Byrne, F.; Davies, G.-L.; Coey, J. M. D.; Volkov, Y.; Gun'ko, Y. K. Small 2010, 6, 247.

(21) Latham, A. H.; Williams, M. E. Accounts of Chemical Research 2008, 41, 411.

(22) Yin, S.-N.; Wang, C.-F.; Yu, Z.-Y.; Wang, J.; Liu, S.-S.; Chen, S. Advanced Materials, 23,

2915.

(23) Vokoun, D.; Beleggia, M.; Heller, L. k.; Å ittner, P. Journal of Magnetism and Magnetic Materials 2009, 321, 3758.

(24) Park, S. Y.; Handa, H.; Sandhu, A. Nano Letters 2009, 10, 446.

(25) Hauck, T. S.; Giri, S.; Gao, Y.; Chan, W. C. W. Advanced Drug Delivery Reviews 2010, $62,438$.

(26) Henriquez, R. R.; Ito, T.; Sun, L.; Crooks, R. M. Analyst 2004, 129, 478.

(27) Sexton, L. T.; Horne, L. P.; Martin, C. R. Molecular BioSystems 2007, 3, 667.

(28) Dekker, C. Nat Nano 2007, 2, 209.

(29) Steinbock, L. J.; Stober, G.; Keyser, U. F. Biosensors and Bioelectronics 2009, 24,

2423.

(30) Willmott, G. R.; Broom, M. F.; Jansen, M. L.; Young, R. M.; Arnold, W. M. Molecular and nano-tubes (Springer, Berlin, 2011). Tunable elastomeric pores 2011, Ch 7, 209.

(31) Willmott, G. R.; Vogel, R.; Yu, S. S. C.; Groenewegen, L. G.; Roberts, G. S.; Kozak, D.; Anderson, W.; Trau, M. Journal of Physics: Condensed Matter 2010, 22, 454116.

(32) Vogel, R.; Willmott, G.; Kozak, D.; Roberts, G. S.; Anderson, W.; Groenewegen, L.; Glossop, B.; Barnett, A.; Turner, A.; Trau, M. Analytical Chemistry 2011, 83, 3499. 
(33) Ang, Y. S.; Yung, L.-Y. L. ACS Nano 2012.

(34) Rowe, W.; Platt, M.; Day, P. J. R. Integrative Biology 2009, 1, 53.

(35) Levy-Nissenbaum, E.; Radovic-Moreno, A.; Wang, A.; Langer, R.; Farokhzad, O. Trends Biotechnol 2008, 26, 442

(36) Mayer, G. Angew Chem Int Ed Eng/ 2009, 48, 2672

(37) Fang, X.; Tan, W. Acc Chem Res 2009, 43, 48

(38) Ellington, A. D.; Szostak, J. W. Nature 1990, 346, 818.

(39) Tuerk, C.; Gold, L. Science 1990, 249, 505

(40) Rowe, W.; Wedge, D. C.; Platt, M.; Kell, D. B.; Knowles, J. Bioinformatics 2010, 26,

2145.

(41) Rowe, W.; Platt, M.; Wedge, D. C.; Day, P. J. R.; Kell, D. B.; Knowles, J. D. Physical Biology 2010, 7, 036007.

(42) Knight, C. G.; Platt, M.; Rowe, W.; Wedge, D. C.; Khan, F.; Day, P. J. R.; McShea, A.; Knowles, J.; Kell, D. B. Nucleic Acids Research 2009, 37, e6.

(43) Platt, M.; Rowe, W.; Wedge, D. C.; Kell, D. B.; Knowles, J.; Day, P. J. R. Analytical Biochemistry 2009, 390, 203.

(44) Willmott, G. R.; Parry, B. E. T. Journal of Applied Physics 2011, 109.

(45) Heins, E. A.; Siwy, Z. S.; Baker, L. A.; Martin, C. R. Nano Letters 2005, 5, 1824.

(46) Kozak, D.; Anderson, W.; Vogel, R.; Chen, S.; Antaw, F.; Trau, M. ACS Nano 2012, 6, 6990.

(47) Lan, W.-J.; Holden, D. A.; Liu, J.; White, H. S. The Journal of Physical Chemistry C 2011, 115, 18445.

(48) Lan, W.-J.; Holden, D. A.; Zhang, B.; White, H. S. Analytical Chemistry 2011, 83, 3840.

(49) Willmott, G. R.; Platt, M.; Lee, G. U. Biomicrofluidics 2012, 6, 014103.

(50) Vogel, R.; Anderson, W.; Eldridge, J.; Glossop, B.; Willmott, G. R. Analytical chemistry 2011, 84, 3125.

(51) Somerville, J. A.; Willmott, G. R.; Eldridge, J.; Griffiths, M.; McGrath, K. M. Journal of Colloid and Interface Science 2013, 394, 243.

(52) Anderson, W.; Kozak, D.; Coleman, V. A.; Jämting, Å. K.; Trau, M. Journal of Colloid and Interface Science.

(53) Platt, M.; Rowe, W.; Knowles, J.; Day, P. J.; Kell, D. B. Integrative Biology 2009, 1, 116.

(54) Cox, J.; Rudolph, P.; Ellington, A. Biotechnol Prog 1998, 14, 845

(55) Pabinger, I.; Ay, C. Arteriosclerosis, Thrombosis, and Vascular Biology 2009, $29,332$.

(56) Chiu, T.-C.; Huang, C.-C. Sensors 2009, 9, 10356.

(57) Macaya, R. F.; Schultze, P.; Smith, F. W.; Roe, J. A.; Feigon, J. Proceedings of the National Academy of Sciences 1993, 90, 3745.

(58) de Puig, H.; Federici, S.; Baxamusa, S. H.; Bergese, P.; Hamad-Schifferli, K. Small 2011, 7, 2477.

(59) Hasegawa, H.; Taira, K.-i.; Sode, K.; Ikebukuro, K. Sensors 2008, 8, 1090.

(60) Katilius, E.; Flores, C.; Woodbury, N. W. Nucleic Acids Research 2007, 35, 7626.

(61) Cohen-Tannoudji, L.; Bertrand, E.; Baudry, J.; Robic, C.; Goubault, C.; Pellissier, M.; Johner, A.; Thalmann, F.; Lee, N. K.; Marques, C. M.; Bibette, J. Physical Review Letters 2008, 100, 108301.

(62) Baudry, J.; Rouzeau, C.; Goubault, C.; Robic, C.; Cohen-Tannoudji, L.; Koenig, A.; Bertrand, E.; Bibette, J. Proceedings of the National Academy of Sciences 2006, 103, 16076.

(63) Davis, S. Biacore Application notes 3051994.

(64) Bock, L. C.; Griffin, L. C.; Latham, J. A.; Vermaas, E. H.; Toole, J. J. Nature 1992, 355, 564.

(65) Pasternak, A.; Hernandez, F. J.; Rasmussen, L. M.; Vester, B.; Wengel, J. Nucleic Acids Research 2011, 39, 1155. 
(66) Tasset, D. M.; Kubik, M. F.; Steiner, W. Journal of Molecular Biology 1997, 272, 688.

For TOC only 


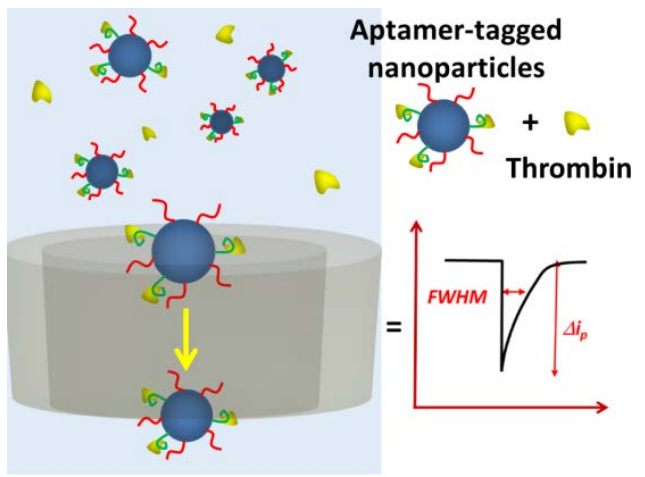

